\title{
Quantum Stabilizer Codes Can Realize Access Structures Impossible by Classical Secret Sharing
}

\author{
Ryutaroh Matsumoto \\ Dept. of Information and Communications Engineering \\ Tokyo Institute of Technology, Japan. \\ Email: ryutaroh@rmatsumoto.org
}

January 11, 2017

\begin{abstract}
We show a simple example of a secret sharing scheme encoding classical secret to quantum shares that can realize an access structure impossible by classical information processing with limitation on the size of each share. The example is based on quantum stabilizer codes.
\end{abstract}

\section{Introduction}

Secret sharing (SS) [12] is a cryptographic scheme to encode a secret to multiple shares being distributed to participants, so that only qualified sets of participants can reconstruct the original secret from their shares. Traditionally both secret and shares were classical information (bits). Several authors [3, 5, 13] extended the traditional SS to quantum one so that a quantum secret can be encoded to quantum shares. The family of qualified sets are called the access structure. In this note, we study perfect secret sharing, in which every unqualified set has absolutely no information about secret. An $(n, k)$ threshold secret sharing scheme distributes secret to $n$ participants and enables $k$ or more participants to reconstruct the secret while $k-1$ or less participants have no information about the secret. 
Cascudo et al. 22 studied what kind of access structures can be realized with small share size $\bar{q}$, and proved that $\bar{q} \geq n-k+2$ as a special case of their main result for secret sharing schemes with classical secret and classical shares, where $\bar{q}$ is the average of the cardinalities of all share sets. This means that there cannot be a $(3,5)$ threshold scheme distributing 1-bit secret to 5 participants receiving 1-bit shares, because $\bar{q}=2, n=5$ and $k=3$ in that case.

For secret sharing schemes with quantum secret and quantum shares, it has been observed that quantum error-correcting codes (QECC) can be used for the purpose [3, 5, 6, 8, 13]. A quantum secret is simply encoded by a QECC into $n$ quantum systems, which are distributed to $n$ participants. A qualified set of participants can reconstruct the quantum secret by the erasure-correcting procedure with treating missing shares as erasures. The dimension of a quantum share corresponds to the size of a classical share, for example, a qubit corresponds to a classical share of size 2, i.e., a bit.

The above QECC can also distribute classical secret, just by regarding classical secret (random variable) as quantum information (density matrix). The purpose of this note is to show that the well-known binary stabilizer QECC can realize a $(3,5)$ secret sharing scheme distributing 1-bit classical secret to 5 participants receiving 1-qubit of quantum shares. If we regard a qubit as an equivalent information unit to a bit, it means $q=2, n=5$ and $k=3$ that cannot be realized by purely classical information processing [2]. The rest of this note mainly consists of an analysis of the access structure of a secret sharing scheme constructed from a binary stabilizer QECC.

We note that a $(3,5)$ scheme was already realized by [7, Section IV.B] with qubit shares, but the QECC based secret sharing has a much simpler protocol because it just uses an encoding procedure and the erasure decoding procedure of the QECC (or the recently proposed unitary reconstruction procedure [9]).

\section{Access Structure of the Secret Sharing Scheme}

\subsection{Secret Sharing Scheme and Qualified Sets}

We consider a binary stabilizer QECC encoding 1 qubit to 5 qubit [4, Section 3.3]. Qubit $|0\rangle$ is encoded to

$$
|\psi(0)\rangle
$$




$$
\begin{aligned}
= & |00000\rangle+|10010\rangle+|01001\rangle+|10100\rangle \\
& +|01010\rangle-|11011\rangle-|00110\rangle-|11000\rangle \\
& -|11101\rangle-|00011\rangle-|11110\rangle-|01111\rangle \\
& -|10001\rangle-|01100\rangle-|10111\rangle+|00101\rangle,
\end{aligned}
$$

and $|1\rangle$ is encoded to

$$
\begin{aligned}
& |\psi(1)\rangle \\
= & |11111\rangle+|01101\rangle+|10110\rangle+|01011\rangle \\
& +|10101\rangle-|00100\rangle-|11001\rangle-|00111\rangle \\
& -|00010\rangle-|11100\rangle-|00001\rangle-|10000\rangle \\
& -|01110\rangle-|10011\rangle-|01000\rangle+|11010\rangle .
\end{aligned}
$$

To share classical bit 0 (resp. 1), each qubit in $|\psi(0)\rangle$ (resp. $|\psi(1)\rangle$ is distributed to each participants. In error correction, an erasure means a (quantum or classical) error with known location. The above QECC can correct up to two erasures. By regarding classical bit 0 as quantum information $|0\rangle\langle 0|$ and 1 as $|1\rangle\langle 1|$, we can see that three or more participants can reconstruct the classical secret.

\subsection{Unqualified Sets}

In order to clarify the access structure of the above secret sharing scheme, we have to study what can be known to two or less participants. One can use the Holevo information [10] between classical information (probability distribution) and quantum information (density matrix) to study such a problem. Let $J \subset\{1, \ldots, 5\}$ be a set of shares (participants), and $\rho_{s}^{J}$ be the quantum state of shares in $J$ corresponding to secret $s \in\{0,1\}$. Let $q_{0}$ and $q_{1}$ be a probability distribution of the secret $s$ on $\{0,1\}$. The Holevo information is defined by

$$
I(J)=S\left(q_{0} \rho_{0}^{J}+q_{1} \rho_{1}^{J}\right)-\left(q_{0} S\left(\rho_{0}^{J}\right)+q_{1} S\left(\rho_{1}^{J}\right)\right) .
$$

If $I(J)=0$ then outcomes (interpreted as random variables) of any measurement of the share set $J$ are statistically independent of the secret $s$.

The secret sharing scheme considered here can also be regarded as sharing quantum secret $\alpha_{0}|0\rangle+\alpha_{1}|1\rangle$ producing the quantum shares $\alpha_{0}|\psi(0)\rangle+$ $\alpha_{1}|\psi(1)\rangle$. If $|J| \leq 2$ then $\bar{J}=\{1, \ldots, 5\} \backslash J$ can reconstruct the quantum secret $\alpha_{0}|0\rangle+\alpha_{1}|1\rangle$, as the minimum distance of this QECC is 3 [4]. By [3, 5], 
this implies that $J$ has absolutely no information about the quantum secret $\alpha_{0}|0\rangle+\alpha_{1}|1\rangle$. By [11, Theorem 2], we see $I(J)=0$, which means that $J$ has no information about the classical secret $s$, in other words, any measurement on $J$ gives outcomes statistically independent of $s$.

So we can see that this QECC is a $(3,5)$ scheme distributing one qubit to each participant as a share. If each qubit in a share is replaced by a bit, then a $(3,5)$ scheme cannot be realized [2]. Thus this simple example demonstrates that an impossible access structure given a limitation on the size of shares within classical information processing sometimes becomes realizable by quantum information processing, especially by quantum stabilizer codes. This is a stark contrast to the fact that quantum secret sharing constructed from the CSS QECC [1, 14] has an access structure whose qualified sets are always qualified [8] in the corresponding classical secret sharing.

\section{Acknowledgment}

The author would like to thank Prof. Tomohiro Ogawa for helpful discussion. This research is partly supported by the JSPS Grant No. 26289116.

\section{References}

[1] A. R. Calderbank and P. W. Shor, Good quantum error-correcting codes exist. Phys. Rev. A, 54(2)1098-1105, Aug. 1996.

[2] I. Cascudo, R. Cramer, and C. Xing. Bounds on the threshold gap in secret sharing and its applications. IEEE Trans. Inform. Theory, 59(9):5600-5612, Sept. 2013.

[3] R. Cleve, D. Gottesman, and H.-K. Lo. How to share a quantum secret. Phys. Rev. Lett., 83(3):648-651, July 1999, quant-ph/9901025.

[4] D. Gottesman. Stabilizer Codes and Quantum Error Correction. PhD thesis, California Institute of Technology, May 1997, arXiv:quant-ph/9705052.

[5] D. Gottesman. Theory of quantum secret sharing. Phys. Rev. A, 61(4):042311, Mar. 2000, quant-ph/9910067.

[6] A. Marin and D. Markham. Equivalence between sharing quantum and classical secrets and error correction. Phys. Rev. A, 88(4):042332, 2013.

[7] D. Markham and B. C. Sanders. Graph states for quantum secret sharing. Phys. Rev. A, 78(4):042309, 2008. 
[8] R. Matsumoto. Coding theoretic construction of quantum ramp secret sharing. 2014, arXiv:1405.0149v5.

[9] R. Matsumoto. Unitary reconstruction of secret for stabilizer based quantum secret sharing. 2017, arXiv:1701.02054.

[10] M. A. Nielsen and I. L. Chuang. Quantum Computation and Quantum Information. Cambridge University Press, Cambridge, UK, 2000.

[11] T. Ogawa, A. Sasaki, M. Iwamoto, and H. Yamamoto. Quantum secret sharing schemes and reversibility of quantum operations. Phys. Rev. A, 72(3):032318, Sept. 2005, arXiv:quant-ph/0505001.

[12] A. Shamir. How to share a secret. Comm. ACM, 22(11):612-613, Nov. 1979.

[13] A. D. Smith. Quantum secret sharing for general access structures. Jan. 2000, quant-ph/0001087.

[14] A. M. Steane, Multiple particle interference and quantum error correction. Proc. Roy. Soc. London Ser. A, 452(1954)2551-2577, 1996. 